\title{
Different evolutionary stages in S235A-B
}

\author{
Marcello Felli ${ }^{1}$, Fabrizio Massi ${ }^{1}$ and Riccardo Cesaroni ${ }^{1}$ \\ ${ }^{1}$ INAF Osservatorio Astrofisico di Arcetri, Largo Enrico Fermi 5, I-50125 Firenze, Italy \\ email: felli@arcetri.astro.it
}

\begin{abstract}
The star forming region S235A-B has been studied at high resolution with radio (IRAM Interferometer and VLA) and infrared (JCMT and Spitzer) observations. The region was mapped in $\mathrm{HCO}^{+}, \mathrm{C}^{34} \mathrm{~S}, \mathrm{H}_{2} \mathrm{CS}, \mathrm{SO}_{2}$ and $\mathrm{CH}_{3} \mathrm{CN}$ as well as in the 1.2 and 3.3 continuum, in the cm continuum at $6,3.6,1.3$ and $0.7 \mathrm{~cm}$ and in the $22 \mathrm{GHz}$ water maser line, in the far infrared at 450 and $850 \mu \mathrm{m}$ and in the mid infrared from 3.6 to $8 \mu \mathrm{m}$. Finally, use was made of the Medicina water maser patrol, from 1987 to 2005, to study the maser variability.
\end{abstract}

Keywords. Stars: formation - ISM: individual objects: S235A-B - ISM: jets and outflows Radio continuum: ISM - Masers

\section{Discussion}

S235A is a classical HII region, well resolved and with a very good match between radio and infrared images. To the south of it, molecular and continuum mm observations as well as infrared observations unambiguously reveal the presence of a newly formed YSO placed in between the S235A and S235B: a molecular core and an unresolved mm source are centred on a water maser, with indication of mass infall onto the core. Two bipolar outflows detected in $\mathrm{HCO}^{+}$and a jet originate from the same position (Felli et al. 1997; Felli et al. 2004).

No cm radio continuum emission is detected either from the compact molecular core or from the jet-like structure, suggesting emission from dust in both cases. A weak evidence is found from $\mathrm{C}^{34} \mathrm{~S}$ observations for a molecular rotating disk perpendicular to the main bipolar outflow. The derived parameters indicate that the YSOs is an intermediate luminosity object in a very early evolutionary phase. Its main source of energy could come from gravitational infall thus making of this YSO a rare link between the earliest evolutionary phases of massive stars and low mass protostars of Class 0-I.

Two compact radio continuum sources (VLA-1 and VLA-2) and three separate maser spots were found. VLA-1 coincides with one of the maser spots and with an IR source (M1). VLA-2 lies towards S235B and represents the first radio detection from this peculiar nebula. The two other maser spots coincide with the rotating disk perpendicular to the bipolar molecular outflow. The Spitzer images reveal a red object towards the molecular core which is the most viable candidate for the embedded YSO.

The picture emerging from all these data shows the extreme complexity of a small star forming region where a more evolved region (S235A) may trigger the formation of a younger one (Felli et al. 2006).

\section{References}

Felli, M., Testi, L., Valdettaro, R., \& Wang, J.-J. 1997, A\&3A 320, 594

Felli, M., Massi, F., Navarrini, A. et al. 2004, A $E A$ 420, 553

Felli, M., Massi, F., Robberto, M., \& Cesaroni, R. 2006, A\& A 453, 911 